doi:10.4149/neo_2014_027

\title{
Prognostic significance of TMPRSS4 in gastric cancer
}

\author{
H. SHENG, W. SHEN* ${ }^{*}$ J. ZENG, L. XI, L. DENG \\ Department of Oncology, Jiangyin Hospital Affiliated to Medical College of Southeast University, Jiangyin 214400, China \\ ${ }^{*}$ Correspondence: jyshenweisheng@yeah.net
}

Received May 29, 2013 / Accepted July 4, 2013

\begin{abstract}
Transmembrane protease, serine 4 (TMPRSS4), is a novel type of II transmembrane serine protease that is highly expressed in multiple cancers. However the expression pattern and clinical significance of TMPRSS4 in gastric cancer (GC) remain unclear. We aimed to investigate the clinical and prognostic importance of TMPRSS4 in GC. We performed an analysis of TMPRSS4 expression in 200 consecutive GC patients by immunohistochemistry. Based on TMPRSS4 positivity, the GC tissues were divided into TMPRSS4-positive group and TMPRSS4-negative group. The relationships between TMPRSS4 expression and clinicopathological parameters or prognosis were explored. The positivity of TMPRSS4 expression was significantly higher in GC tissues than in adjacent normal tissues ( $44.5 \%$ versus $9.5 \%, \mathrm{P}<0.001)$. TMPRSS4 positivity was significantly correlated with depth of invasion, lymph node metastasis, and vessel invasion (all $\mathrm{p}<0.01$ ). Survival analysis indicated that the TMPRSS4positive group showed poorer survival than the TMPRSS4-negative group $(p<0.01)$. In terms of clinical stages, significantly different TMPRSS4 positivity was found only in stages II and III. Multivariate analysis showed that TMPRSS4 expression was an independent prognostic factor $(\mathrm{p}=0.013)$. Our data suggest that TMPRSS4 positivity is associated with GC invasion and lymph node metastasis. We propose TMPRSS4 expression as an indicator of poor prognosis in GC patients.
\end{abstract}

Key words: gastric cancer, TMPRSS4, prognosis, biomarker

Gastric cancer (GC) is one of the most common malignancies and the second leading cause of cancer death worldwide [1]. Despite rapid advances in current treatment protocols incorporating chemoradiation as well as surgical procedures, GC continues to be a fatal disease with majority of cases presenting in advanced stages and we can only recommend only palliative therapy [2]. The clinical stage at diagnosis and the curative surgery are the most important for GC treatment. However, nodal invasion, distant metastasis and local relapses frequently occur even after comprehensive therapy. Metastasis is the main cause of death, but the mechanism underlying metastatic process in GC is very complex and incompletely understood [3-4]. Hence, it is important for clinicians to identify well-characterized biomarkers that would predict metastasis and prognosis of GC patients, in order to facilitate therapeutic intervention.

Tumor cells utilize numerous cellular or biochemical mechanisms to complete metastasis [5]. Type II transmembrane serine proteases (TTSPs) have reported to be upregulated during the tumor growth and progression [6]. Transmembrane protease, serine 4 (TMPRSS4) belongs to a family of membrane-type serine proteases. Several studies have indicated
mRNA expression of TMPRSS4 is increased in pancreatic, colorectal, and thyroid cancers [7-9]. Recently, two studies have shown that high levels of TMPRSS4 are associated with poor prognosis in breast cancer and non-small cell lung cancer patients [10-11].

However, the expression pattern of TMPRSS4 and its clinical significance in GC have not been explored to date. In this study, we aimed to investigate the expression pattern and clinicopathological implications of TMPRSS4 in GC. Here we provided evidence that TMPRSS4 expression was significantly upregulated in GC tissues, compared to the adjacent normal tissues and was significantly associated with lymph node metastasis and worse survival of GC patients.

\section{Patients and methods}

Patients. Data came from 200 consecutive GC patients who received resection at the Department of Oncology, Jiangyin Hospital Affiliated to Medical College of Southeast University from January 2006 to January 2012. The patients consisted of 137 males and 63 females. No patients received chemotherapy before operation. All patients received $\mathrm{R} 0$ resection with more 
than D1 lymph node dissection (Table 1). This study was approved by the Ethical Committee of the Medical College of Southeast University, and informed consent was obtained from all participants.

TMPRSS4 expression by immunohistochemistry and western blot. Immunohistochemistry was carried out following manufacturer instructions (DAKO). And paraffinembedded sections $(4 \mu \mathrm{m})$ were prepared on silane-coated slides (Sigma, USA) and deparaffinized and incubated with $3 \% \mathrm{H}_{2} \mathrm{O}_{2}$ in methanol for 10 minutes to block endogenous tissue peroxidase. The antigen was retrieved at $95^{\circ} \mathrm{C}$ for 20 minutes in $10 \mathrm{mM}$ sodium citrate buffer. The slides were then incubated with rabbit anti-TMPRSS4 antibody (1:50, Abcam, USA) at $4^{\circ} \mathrm{C}$ overnight. After incubation with biotinylated secondary antibody for 30 minutes at room temperature, the slides were incubated with streptavidin-peroxidase complex for 30 minutes at room temperature. Immunostaining was developed by using streptavidin peroxidase 3,3' -diaminobenzidine-chromogen detection. We used the rabbit IgG isotope controls, which showed negative staining.

Furthermore, we used western blot to confirm the TMPRSS4 expression in the GC tissue. Western blot was carried out with rabbit polyclonal anti-TMPRSS4 antibody (1:2000; Abcam, USA) or GAPDH (internal control). An HRP-conjugated anti-rabbit IgG antibody was used as the secondary antibody (Santa Cruz, USA). Signals were detected using enhanced chemiluminescence reagents (Pierce).

Evaluation of TMPRSS4 positivity in GC. All slides were scored by two independent investigators blinded to this study. The interpretation criteria were described previously [12]. Briefly, the immunoreactivity was graded as: +++ (score 3$) ;++$ (score 2$) ;+($ score 1 ) and - (score 0$)$. Score $0-1$ was regarded as TMPRSS4 negative and score 2-3 as TMPRSS4 positive.

Table 1. Information of Patients

\begin{tabular}{llc}
\hline Item & features & $\mathrm{N}$ \\
\hline Gender & Male & 137 \\
& Female & 63 \\
Age & & $62(41-85)$ \\
Operation & Total gastrectomy & 67 \\
& Distal gastrectomy & 108 \\
& Proximal gastrectomy & 25 \\
Stage & I & 69 \\
& II & 29 \\
& III & 54 \\
& IV & 48 \\
Histology & Differentiated & 87 \\
& Undifferentiated & 113 \\
TMPRSS4 positivity & Yes & 89 \\
& No & 111 \\
\hline
\end{tabular}

Patients were divided into two groups according to TMPRSS4 positivity. The correlations between clinicopathological features and TMPRSS4 expression were investigated.

Statistical analysis. Statistical analysis was performed using the Chi-square ( $\mathrm{x}^{2}$-test) test by SPSS17.0 software. Cumulative survival curves were analyzed by the Kaplan-Meier method and statistical significance was calculated using log-rank test. The Cox proportional hazard model was used in the multivariate analysis to determine prognostic factors. $\mathrm{P}<0.05$ was considered significant.

\section{Results}

TMPRSS4 protein staining in GC. GC samples and normal adjacent area were analyzed. TMPRSS4 positive staining was mainly observed in the cytoplasm of gastric cancer cells as dark brown granules (Figure 1) by immunohistochemistry. Out of all the 200 tissues, we found that the positive rate of TMPRSS4 is obviously higher in GC tissues than in paired normal adjacent tissues ( $44.5 \%$ versus $9.5 \%, \mathrm{P}<0.001$ ).

Clinicopathological features and TMPRSS4 positivity in GC. The correlation between TMPRSS4 positivity and clinicopathological features relating to patient outcome was analyzed. TMPRSS4 positivity significantly correlated with deeper tumor invasion $(\mathrm{p}<0.01)$, lymph node metastasis $(\mathrm{p}<0.01)$, lymphatic invasion $(\mathrm{p}<0.05)$, and venous invasion $(\mathrm{p}<0.05)$. There was no correlation between tumor histology and TMPRSS4 expression (Table 2). The five-year survival rate of the TMPRSS4-positive group was significantly lower than that of the TMPRSS4-negative group ( $41 \%$ vs $72 \%$, $\mathrm{p}=0.014$ ) (Figure 2).

TMPRSS4 positivity in stage GC II and III. In terms of GC stages, we only found significant differences in TMPRSS4

Table 2. Correlation between clinical features and TMPRSS4 positivity

\begin{tabular}{llccl}
\hline Clinical features & & TMPRSS4 & positivity & p value \\
\hline \multirow{3}{*}{ Gender } & Male & Yes n=89 & No n $=111$ & \\
\multirow{4}{*}{ Tumor depth } & Female & 38 & 79 & N.S. \\
& T1 & 23 & 42 & \\
& T2 & 30 & 38 & $\mathrm{p}<0.01$ \\
& T3 & 36 & 30 & \\
Nodal involvement & Yes & 44 & 40 & $\mathrm{p}<0.05$ \\
& No & 45 & 71 & \\
Lymphatic invasion & Yes & 52 & 59 & $\mathrm{p}<0.01$ \\
& No & 37 & 52 & \\
Venous invasion & Yes & 48 & 41 & $\mathrm{p}<0.01$ \\
& No & 41 & 70 & \\
Histology & Differentiated & 39 & 50 & N.S. \\
& Undifferentiated & 50 & 61 & \\
\hline \multirow{5}{*}{} & & & &
\end{tabular}




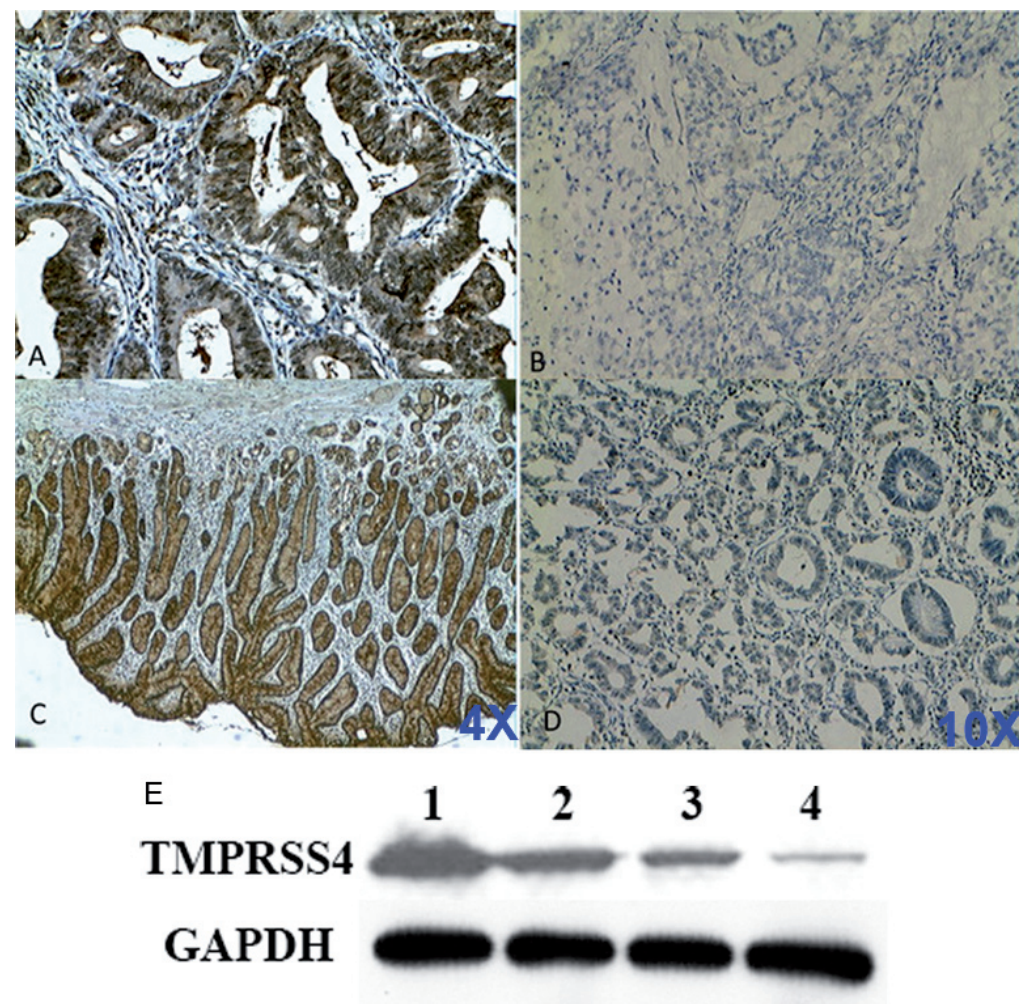

Figure 1. Immunoreactivity for TMPRSS4 protein in gastric cancer (GC). Tissue sections were stained for TMPRSS4 using anti-TMPRSS4 antibody followed by biotinylated secondary antibody incubation and streptavidin peroxidase $3,3^{\prime}$-diaminobenzidine-chromogen detection. The immunoreactivity staining was considered to be dark brown. Immunohistochemical staining of TMPRSS4 in gastric carcinoma (A, B) and adjacent noncancerous (C, D) tissues. A and C. TMPRSS4 positive expression by using the anti-TMPRSS4 antibody; B and D. TMPRSS4 negative expression by using the rabbit IgG isotope. E. Western blot was performed to confirm the higher TMPRSS4 expression in the GC tissue; The GC tissue sample and the corresponding adjacent noncancerous tissue obtained from the same patient. Lanes 1-2: gastric carcinoma, Lanes 3-4: adjacent noncancerous tissues. Tissue sample in lane 1 and 3 was obtained from the same patient, and it was the same with lane 2 and 4.

positivity in stage II and III patients ( $\mathrm{p}=0.0071)$ (Figure 3 ). Other clinical features, including age, sex, depth of invasion, nodal involvement and TMPRSS4 positivity which affect patient outcome by univariate analysis were evaluated by multivariate analysis. As a result, TMPRSS4 expression was a significant prognostic factor in addition to the depth and nodal involvement (Table 3).

\section{Discussion}

GC-related deaths associate with the early metastasis and strong invasion, so it is very important to estimate the malignant degree and invasion tendency of cancer in order to make proper treatment decision. In the present study, we used immunohistochemistry to analyze the expression of TMPRSS4 in 200 consecutive GC patients from January 2006 to January 2012. The results showed that TMPRSS4 positivity was significantly higher in GC tissues compared with normal gastric tissues. In addition, high expression levels of TMPRSS4 in GC lesions were associated with depth of invasion, lymph node metastasis, vessel invasion and TNM stage II-III. Importanly, TMPRSS4 upregulation was an independent prognostic factor in GC. Therefore, TMPRSS4 could be of great value as a diagnostic marker and therapeutic target for GC.

Despite limited evidence of TMPRSS4 involvement in GC, several studies have identified a relationship between TMPRSS4 expression and tumor progression and prognosis. Kebebew et al. reported that TMPRSS4 mRNA expression

Table 3. Univariate and multivariate analysis of survival with clinical features

\begin{tabular}{lcccc}
\hline Factors & Univariate & \multicolumn{3}{c}{ Multivariate } \\
\hline & $\mathrm{p}$ value & $\mathrm{p}$ value & Hazard ratio & $95 \% \mathrm{CI}$ \\
Age & $<0.01$ & $<0.01$ & 0.34 & $0.17-0.63$ \\
Gender & $<0.05$ & N.S. & 0.90 & $0.8-2.2$ \\
TMPRSS4 & $<0.01$ & $<0.05$ & 0.47 & $0.26-0.84$ \\
Tumor depth & $<0.01$ & $<0.01$ & 0.42 & $0.23-0.79$ \\
Nodal involvement & $<0.01$ & $<0.01$ & 0.24 & $0.11-0.54$ \\
Tumor length & $<0.05$ & $<0.05$ & 0.3 & $0.24-0.93$ \\
\hline
\end{tabular}




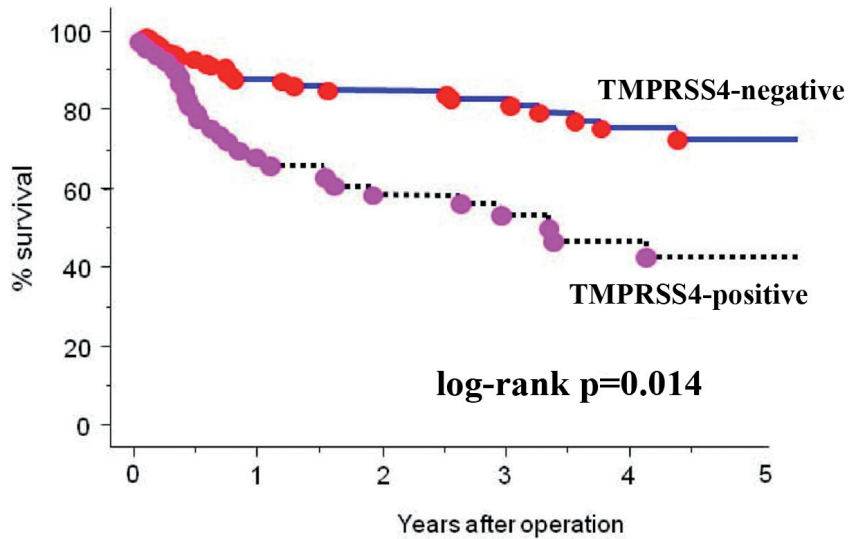

Figure 2 For gastric cancer (GC) patients, survival rate of the TMPRSS4positive group was significantly worse than that of the TMPRSS4-negative group $(41 \%$ vs $72 \%, \mathrm{p}=0.014)$.

was higher in malignant than in benign thyroid neoplasms and could improve the diagnostic accuracy of fine needle aspiration biopsy [13]. Jung and colleagues showed that TMPRSS4 promoted invasion, migration and metastasis of human tumor cells by facilitating an epithelial-mesenchymal transition [14]. Subsequebt data show that TMPRSS4 induces invasion and epithelial-mesenchymal transition through upregulation of integrin alpha5 and its signaling pathways [15]. Recent studies indicate that overexpression of TMPRSS4 is significantly associated with reduced overall survival of patients with non-small cell lung cancer and breast cancer and may represent a potential therapeutic target $[10-11,16]$. However, to date, there is no report to clarify the prognostic impact of TMPRSS4 expression in GC. Therefore, this is the first report to determine the correlation between TMPRSS4 expression and poor prognosis and other clinical factors in GC. Our results showed that TMPRSS4 was upregulated in gastric cancer tissues compared with noncancerous tissues and the positivity of TMPRSS4 in GC affected clinical features directly.

With respect to survival analysis, our results showed in patients with stage II and III GC, the survival rate for those with positive TMPRSS4 expression was significantly lower than that of patients with negative expression. Multivariate analysis suggested that the depth of invasion, lymph node metastasis and TMPRSS4 up-regulation were independent prognostic indicators for GC.

In conclusion, our data showed that GC patients had overexpression of TMPRSS4, which was associated with an aggressive clinical features and poor survival. Therefore, TMPRSS4 may play a significant role in the progression of GC consistent with previous data on other cancers. In addition, such information may provide novel therapeutic and prognostic possibilities for treating GC and improving patient outcome. The roles of TMPRSS4 in cancer merit further prospective analysis.

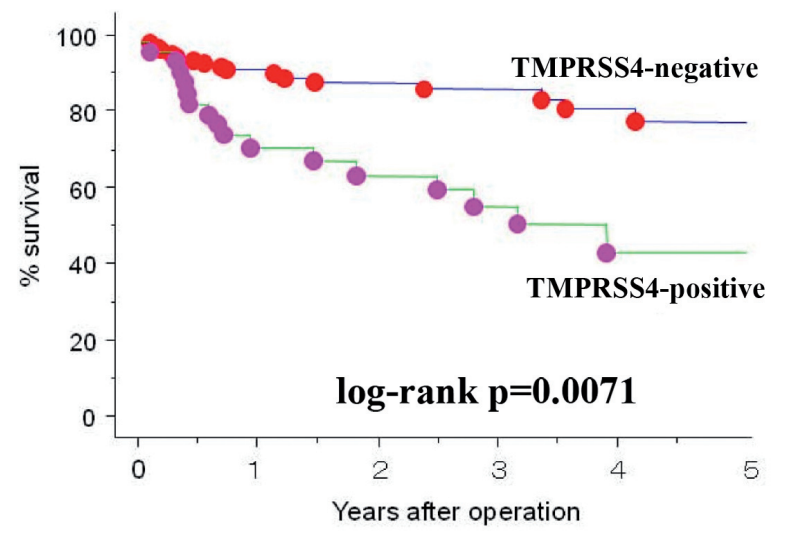

Figure 3 Significant prognostic difference of TMPRSS4 positivity in stage II and III gastric cancer (GC) patients $(\mathrm{p}=\mathbf{0 . 0 0 7 1})$.

\section{References}

[1] FERLAY J, SHIN HR, BRAY F, FORMAN D, MATHERS C, et al. Estimates of worldwide burden of cancer in 2008: GLOBOCAN 2008. Int J Cancer 2010;127: 2893-2917. http://dx.doi. org/10.1002/ijc.25516

[2] BLUM MA, TAKASHI T, SUZUKI A, AJANI JA. Management of localized gastric cancer. J Surg Oncol. 2013; 107: 265-270. http://dx.doi.org/10.1002/jso.23183

[3] ZAGOURI F, PAPADIMITRIOU CA, DiMOPOULOS MA, PECTASIDES D. Molecularly targeted therapies in unresectable-metastatic gastric cancer: a systematic review. Cancer Treat Rev 2010; 37: 599-610. http://dx.doi.org/ 10.1016/j.ctrv.2011.03.007

[4] JANG BG, KIM WH. Molecular pathology of gastric carcinoma. Pathobiology 2011; 78: 302-310. http://dx.doi. org/10.1159/000321703

[5] CURRAN S, MURRAY GI. Matrix metalloproteinases in tumour invasion and metastasis. J Pathol 1999; 189: 300-308. http://dx.doi.org/10.1002/(SICI)10969896(199911)189:3<300::AID-PATH456>3.0.CO;2-C

[6] WEBB SL, SANDERS AJ, MASON MD, JIANG WG. Type II transmembrane serine protease (TTSP) deregulation in cancer. Front Biosci 2011; 16: 539-552. http://dx.doi. org/10.2741/3704

[7] GRUTZMANN R, PILARSKY C, AMMERPOHL O, LUTTGES J, BOHME A, et al. Gene expression profiling of microdissected pancreatic ductal carcinomas using highdensity DNA microarrays. Neoplasma. 2004; 6: 611-622. http://dx.doi.org/10.1593/neo.04295

[8] KEBEBEW E, PENG M, REIFF E, DUH QY, CLARK OH, et al. ECM1 and TMPRSS4 are diagnostic markers of malignant thyroid neoplasms and improve the accuracy of fine needle aspiration biopsy. Ann Surg 2005; 242: 353-361.

[9] RIKER AI, ENKEMANN SA, FODSTAD O, LIU S, REN S, et al. The gene expression profiles of primary and metastatic melanoma yields a transition point of tumor progression and 
metastasis. BMC Med Genomics. 2008; 1: 13. http://dx.doi. org/10.1186/1755-8794-1-13

[10] LIANG B, WU M, BU Y, ZHAO A, XIE F. Prognostic value of TMPRSS4 expression in patients with breast cancer. Med Oncol 2013; 30: 497. http://dx.doi.org/10.1007/s12032-013-0497-8

[11] NGUYEN TH, WEBER W, HAVARI E, CONNORS T, BAGLEY RG, et al. Expression of TMPRSS4 in non-small cell lung cancer and its modulation by hypoxia. Int J Oncol. 2012; 41: 829-838.

[12] WU Q, LI Z, LIN H, HAN L, LIU S, et al. DEK overexpression in uterine cervical cancers. Pathol Int 2008; 58: 378-382. http://dx.doi.org/10.1111/j.1440-1827.2008.02239.x

[13] KEBEBEW E, PENG M, REIFF E, DUH QY, CLARK OH, et al. ECM1 and TMPRSS4 are diagnostic markers of malignant thyroid neoplasms and improve the accuracy of fine needle aspiration biopsy. Ann Surg. 2005; 242: 353-361.
[14. JUNG H, LEE KP, PARK SJ, PARK JH, JANG YS, et al. TMPRSS4 promotes invasion, migration and metastasis of human tumor cells by facilitating an epithelial- mesenchymal transition. Oncogene 2008; 27: 2635-2647. http://dx.doi. org/10.1038/si.onc. 1210914

[15] KIM S, KANG HY, NAM EH, CHOI MS, ZHAO XF, Et al.TMPRSS4 induces invasion and epithelial-mesenchymal transition through upregulation of integrin alpha5 and its signaling pathways. Carcinogenesis 2010; 31: 597-606. http:// dx.doi.org/10.1093/carcin/bgq024

[16] LARZABAL L, NGUEWA PA, PIO R, BLANCO D, SANCHEZ $\mathrm{B}$, et al. Overexpression of TMPRSS4 in non-small cell lung cancer is associated with poor prognosis in patients with squamous histology. Br J Cancer. 2011; 105: 1608-1614. http://dx.doi.org/10.1038/bjc.2011.432 